\title{
Facing the Correct Pole: The Challenge of Orienting Chromosomes for Meiotic Divisions
}

\author{
Karishma Collette and Györgyi Csankovszki \\ Department of Molecular, Cellular and Developmental Biology, University of Michigan, \\ USA
}

\section{Introduction}

Meiosis is a specialized cell division that directs a diploid germ cell to produce haploid gametes (reviewed in Schvarzstein et al, 2010; Sakuno and Watanabe, 2009). Meiosis differs from mitosis, in that one round of DNA replication is followed by two rounds of chromosome segregation. In the first round, meiosis I, homologous chromosomes separate (reductional division), and in the second round, meiosis II, sister chromatids separate (equational division). Meiosis II is similar to mitosis in that in both processes, replicated sister chromatids orient away from each other (are said to be bioriented), and will be separated at the metaphase to anaphase transition. By contrast, meiosis I, represents special challenges to the cell. In the first meiotic division, sister chromatids must face the same pole (are monooriented), and instead homologs, connected by the chiasma, are oriented away from each other toward opposite poles (Fig. 1). This chapter discusses our current understanding of how sister chromatid monoorientation and homolog biorientation are achieved during meiosis I.

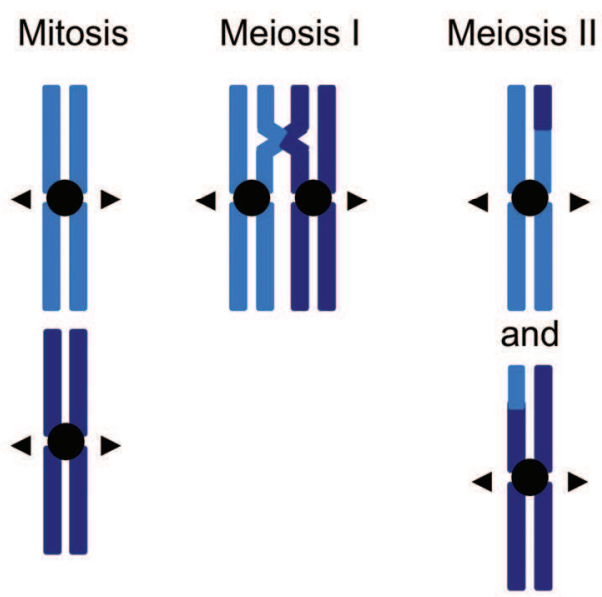

Fig. 1. Orientation of sister chromatids and homologous chromosomes in mitosis and meiosis. Arrowheads indicate the direction chromatids or chromosomes face. 
Organisms with different chromosomal organization deal with this challenge differently. The majority of eukaryotic model organisms studied (such as yeast, flies and mammals) have monocentric chromosomes. Monocentric chromosomes have a single centromere, the region on the chromosomes where the kinetochore assembles. During mitosis and meiosis the kinetochores serve as site of attachment for spindle microtubules (Sakuno and Watanabe, 2009). In monocentric organisms, the centromere is where many meiotic events are coordinated, including orientation of chromosomes. Holocentric chromosomes (in organisms such as the nematode $C$. elegans) lack a localized centromere. On these chromosomes the kinetochore assembles along the entire length of the chromosomes during mitosis and forms cup-like structures encompassing each homolog during meiosis (Schvarzstein et al., 2010) (Fig. 2). Comparisons of the strategies used on chromosomes with such diverse organizational features highlight the common themes and the conserved molecular factors implementing these strategies.

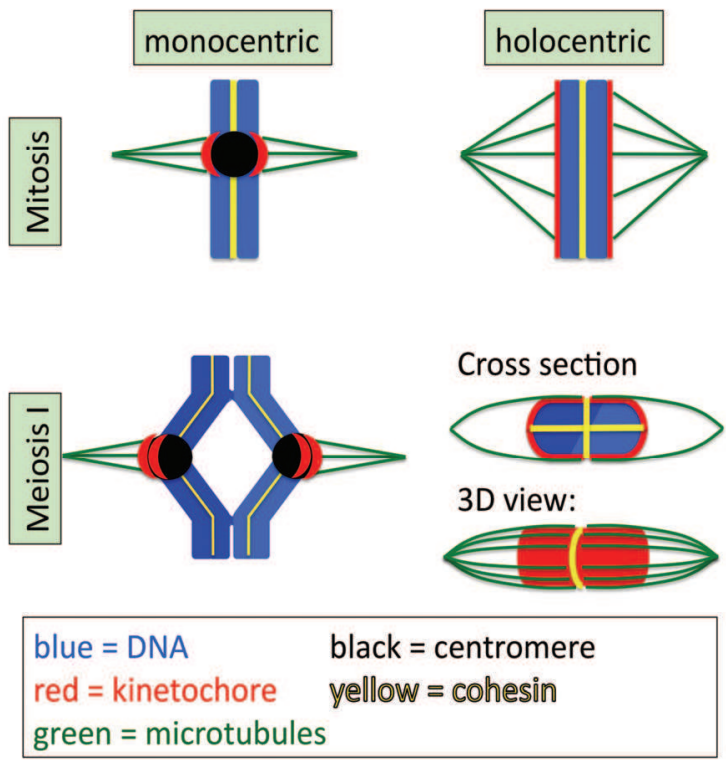

Fig. 2. The organization of kinetochores on monocentric and holocentric chromosomes during mitosis and meiosis I. During mitosis on monocentric chromosomes the kinetochore assembles at the region defined by the centromere. On holocentric chromosomes the kinetochores assemble along the entire length of the chromosomes. During meiosis I on monocentric chromosomes, kinetochores of sister chromatids function as one unit and orient toward the same pole. On holocentric chromosomes the kinetochore cups the volume of the sister chromatid pair.

One crucial player in the process of chromosome orientation is the chromosomal passenger complex (CPC), which monitors and regulates kinetochore-microtubules attachments. The $\mathrm{CPC}$ is composed of Aurora B kinase, inner centromere protein INCENP, Survivin and Borealin/Dasra B. Homologs of CPC subunits have been identified in organisms from yeast to humans. CPC proteins first associate with condensing chromatin at prophase, accumulate 
at the centromere during prometaphase and metaphase, relocate to the spindle midzone at the metaphase-anaphase transition, and finally associate with the midbody during telophase and cytokinesis (Vagnarelli and Earnshaw, 2004). This characteristically dynamic localization of the CPC likely reflects movement of Aurora B to act on different substrates. While on chromosomes, the CPC has important functions in condensin recruitment, facilitation of accurate microtubule-kinetochore attachments, spindle checkpoint function, and cohesin regulation. After the CPC transfers onto the central spindle and midbody, it is needed for the successful execution of cytokinesis (Vagnarelli and Earnshaw 2004).

The Structural Maintenance of Chromosomes (SMC) protein containing complexes condensin and cohesin, influence many aspects of chromosome organization and segregation and play roles in regulating chromosome orientation as well. Condensin is composed of a heterodimer of an SMC2 class and an SMC4 class ATPase protein, and three regulatory subunits referred to as chromosome associated polypeptide (CAP) proteins (Hirano, 2004; Hudson et al., 2009, Losada and Hirano, 2005). Budding and fission yeasts have a single condensin complex, but higher eukaryotes have two: condensin I and II. Condensins I and II differ in the identity of their CAP subunits. Condensin I contains CAP$\mathrm{G}$, -D2, and $-\mathrm{H}$, while condensin II contains CAP-G2, -D3, and -H2. The single yeast condensin is most similar to the condensin I complex of higher eukaryotes. Although their exact molecular contribution to chromosome packaging remains a mystery, condensin complexes are essential for the precise organization and structural integrity of chromosomes (Bhat et al., 1996; Chan et al., 2004; Cobbe et al., 2006; Coelho et al., 2003; Dej et al., 2004; Gerlich et al., 2006; Hagstrom et al., 2002; Hartl et al., 2008; Hirota et al., 2004; Hudson et al., 2003; Lieb et al., 1998; Oliveira et al., 2003, 2005; Ono et al., 2003, 2004; Ribeiro et al., 2009; Samoshkin et al., 2009; Savvidou et al., 2005; Siddiqui et al., 2003; Stear and Roth, 2002; Steffensen et al., 2001; Vagnarelli et al., 2006; Watrin and Legagneux, 2005; Wignall et al., 2003; Yu and Koshland, 2003, 2005). SMC2 and 4 were originally identified as structural components of mitotic chromosomes in Xenopus and chicken cells (Hirano and Mitchison, 1994; Saitoh et al., 1994) and as important regulators of chromosome condensation and segregation in budding and fission yeast (Saka et al., 1994; Strunnikov et al., 1995).

Cohesin contains two SMC ATPase proteins of the SMC1 and SMC3 subclasses, and two regulatory subunits, Scc1 and Scc3 (Hirano, 2002, Jessberger, 2002). In meiosis, Scc1 is replaced by its paralog, Rec8 (Watanabe and Nurse, 1999). Cohesin mediates sister chromatid cohesion from the time they are replicated in $S$ phase until sister chromatids separate at anaphase of mitosis and meiosis (Hirano, 2000; Nasmyth et al., 2001). Condensin and cohesin functions have been recently reviewed elsewhere. In this chapter we will concentrate on their role in regulating chromosome orientation.

\section{Biorientation of sister chromatids in mitosis}

Regulation of kinetochore orientation has been most extensively studied in mitosis. As the principle forces orientating chromosomes in mitosis and meiosis are likely mediated by the same factors, we will begin with a brief review of mitotic chromosome orientation. Mitotic chromosome segregation is preceded by a round of DNA replication, resulting in identical sister chromatids held together by cohesin. At entry into mitosis, or early during mitosis, condensin complexes associate with chromosomes to facilitate their compaction and segregation. In higher eukaryotes, the bulk of cohesin is removed from chromosomes arms 
in prophase, while centromeric cohesin is released at the metaphase to anaphase transition. In yeast, cohesin is removed from chromosomes in a single step, at the metaphase to anaphase transition (Nasmyth, 2002).

Prior to their separation, chromosomes align on the metaphase plate. Chromosome alignment and orientation is complete when all kinetochores are under tension, as a result of two opposing forces. Tension is generated because kinetochore microtubules are pulling sister chromatids toward opposite poles, and this poleward force is opposed by cohesion between sister chromatids in the centromeric region (Hauf and Watanabe, 2004; Sakuno and Watanabe, 2009). Sister kinetochores are arranged back-to-back, an arrangement that naturally facilitates biorientation. Kinetochore-microtubule attachments occur via trial and error, and are stabilized only when tension is established. An ideal attachment in mitosis, with two sister kinetochores attached to microtubules from opposite poles, is called an amphitelic attachment. Erroneous chromosome-spindle attachments, such as attachment of both sister kinetochores to the same pole (syntelic attachment), or attachment of only one sister kinetochore to a pole (monotelic attachment), result in insufficient tension. Merotelic attachment (one sister attached to both poles) could, in principle, establish tension, but must also be corrected (Fig. 3) (Cimini, 2007).
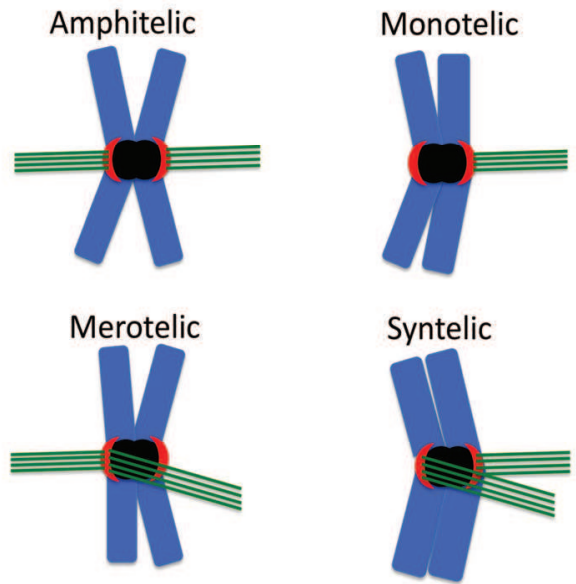

Fig. 3. Various forms of kinetochore-microtubule attachments at mitosis. Chromosomes are shown in blue, centromeres in black, kinetochores in red, and microtubules in green.

During mitosis, the CPC is enriched at the inner centromere where it is perfectly situated to monitor and correct aberrant kinetochore-microtubule attachments. (Tanaka, 2002; Vagnarelli and Earnshaw, 2004; Ruchaud et al. 2007; Watanabe, 2010) CPC depletion drastically disrupts metaphase chromosome alignment and orientation and subsequent anaphase segregation (Biggins et al., 1999; Biggins and Murray, 2001; Carvalho et al., 2003; Cimini et al., 2006; Ditchfield et al., 2003; Hauf et al., 2003, 2007; Honda et al., 2003; Kallio et al., 2002; Knowlton et al., 2006; Lens et al., 2003; Liu et al., 2009; Tanaka et al., 2002; Zhu et al., 2005). Aurora B and the CPC detect inappropriate or reduced tension at sites of incorrect attachment, and facilitate the release of these ill-fated connections. Aurora B appears to assume a more emphatic role in regulating syntely (Hauf, 2003, 2007; Lampson, 2004), but 
merotely is also fixed by the kinase (Cimini et al., 2006; Hauf et al., 2007; Knowlton et al., 2006). Hesperadin (a specific Aurora B inhibitor) treated HeLa cells showed a high incidence of syntelic attachments, and monotelic attachments were reduced six fold compared to control. These results led to a model whereby kinase function is required for the conversion of syntelic into temporary monotelic connections, such that the newly released kinetochore can search for microtubules from the correct pole (Hauf et al., 2003). A study in budding yeast suggests that centromeric INCENP-survivin (another CPC subunit) is a tension sensor that communicates with Aurora B to specifically destroy attachments under aberrant tension (Sandall et al., 2006).

Recent research has suggested that the spatial distribution of Aurora B is key to coupling tension sensing with the stability of kinetochore-microtubule attachments. Aurora B kinase influences microtubule interactions via phosphorylation of various centromeric/kinetochore targets. The phosphorylation status of Aurora B substrates depends on the extent of spatial separation from Aurora B at the inner centromere. Targets experiencing low tension (for example, in a syntelic connection) are physically closer to the kinase at the inner centromere, and undergo phosphorylation, whereas targets subjected to high tension (as seen in a bioriented attachment) are pulled away from Aurora B and escape phosphorylation (Tanaka et al., 2002; Liu et al., 2009).

Aurora B targets include the centromere associated kinesin MCAK (Andrews et al., 2004; Lan et al., 2004) and kinetochore proteins Dam1p, Ndc80p (Cheeseman et al., 2002) and Ndc10p (Biggins et al., 1999). Aurora B activation abolishes incorrect kinetochoremicrotubule attachments by promoting microtubule fiber turnover and disassembly (Lampson et al., 2004; Cimini et al., 2006). Paradoxically, the microtubule-depolymerizing activity of MCAK, a major regulator of microtubule dynamics, is suppressed by Aurora B phosphorylation (Andrews et al., 2004; Lan et al., 2004). Perhaps phosphorylation of MCAK temporarily turns off its depolymerizing activity and supports new attempts at establishing kinetochore-microtubule connections, once incorrect attachments have been resolved (Knowlton et al., 2006; Lan et al., 2004).

Phosphorylation by Aurora B reduces the microtubule binding affinity of its kinetochore substrates (Cheeseman et al., 2006; DeLuca et al., 2006; Ciferri et al., 2008). Phosphorylation by Aurora $\mathrm{B}$ also disrupts subunit interactions within the multiprotein kinetochore and may thus contribute to the elimination of inappropriate kinetochore-microtubule connections (Shang et al., 2003). Thus, phosphorylation of the kinetochore targets of aurora B reduces their affinity for microtubules, causing them to relinquish wrong connections, and seek out fibers coming from the appropriate pole.

The CPC functions not only to detect and dissolve improper attachments, but it also activates the spindle attachment checkpoint (SAC) (Biggins and Murray, 2001; Carvalho et la., 2003; Ditchfield et al., 2003; Lens et al., 2003). SAC is activated in response to insufficient tension or unattached kinetochores. Checkpoint activation delays anaphase onset, so that bipolar attachments can be achieved for all chromosomes.

Tension is only established if the pulling forces of the spindle are counteracted by cohesion between sister centromeres. Centromeric cohesin function is needed for bipolar attachment of sister kinetochores (Sonoda et al., 2001; Tanaka et al., 2000). In fission yeast, cohesin localizes specifically to pericentric regions, and less to the core centromere (Bernard et al., 
2001; Nonaka et al., 2002; Tomonaga et al., 2000). These results support a model in which reduced levels of cohesin at the core centromere allow core regions to open up and assume back-to-back orientation, while pericentric cohesion ensures proper establishment of tension (Sakuno et al., 2009; Sakuno and Watanabe, 2009).

In addition to cohesin, condensin also seems to play a role in ensuring proper biorientation of sisters. In C. elegans, condensin depletion results in disorganized centromeres, and merotelic attachment (Hagstrom et al., 2002; Stear and Roth, 2002). In condensin depleted vertebrate cells, sister kinetochores move closer to each other and they do not face opposite poles anymore (Ono et al., 2004). It is likely that if the underlying chromosome structure is disrupted, especially at the centromeric regions, kinetochore function is perturbed as well.

\section{Chromosomes are restructured extensively in preparation for meiosis}

During meiosis I, sister chromatids orient toward the same pole and paired homologs (called bivalents) held together by the chiasma, become bioriented at metaphase instead (See Fig. 1). Differences in chromosome orientation during mitosis and meiosis could, in principle, be due to differences in the structure of the chromosomes themselves, or differences in the spindle. Micromanipulation experiments support the former possibility (Paliulis and Nicklas, 2000). Meiotic chromosomes placed on a mitotic spindle orient like meiotic chromosomes, and vice versa.

In both monocentric and holocentric organisms, chromosomes are extensively restructured during prophase of meiosis I to facilitate their proper segregation (reviewed in (Page and Hawley, 2003; Schvarzstein et al., 2010). Homologous chromosomes first pair, synapse (form a structure called the synaptonemal complex (SC) along the entire length of the homolog interface), and undergo crossing over, leading to the formation of bivalents. Metaphase chromosome alignment faces two challenges. First, sister kinetochores must be held together and orient toward the same pole. Second, homologs must be oriented away from each. As in mitosis, tension must be established. This tension is mediated by the pulling forces of the spindle microtubules exerted on kinetochores of homologs. Unlike in mitosis, the pulling forces of spindle microtubules are not counterbalanced by cohesion between sister kinetochores, but by the physical linkage of homologs mediated by the chiasma (see Fig. 2). Although research thus far has primarily focused on the importance of the CPC for sister chromatid biorientation in mitosis, recent evidence suggests that its role in destabilizing improper kinetochore-microtubule attachments may be conserved in meiosis as well (see below).

To deal with the special challenges of meiosis, the distribution and/or composition of chromosomal proteins can be different on meiotic chromosomes, as compared to mitosis. In mouse oocytes at metaphase I, condensin II concentrates within the core of individual sister chromatids, while condensin I is enriched at the centromere (Lee et al., 2011). This is in contrast to the mitotic distribution of these complexes. During mitosis in HeLa cells, the two condensin complexes alternate along chromosome axes, with condensin II being enriched at the centromere (Ono et al., 2004) (See Fig. 4). An additional difference between mitosis and meiosis is the subunit composition of cohesin. In meiosis, Rec8 (and other Rec8 paralogs) replace the mitotic paralog Scc1 (Watanabe and Nurse, 1999). 


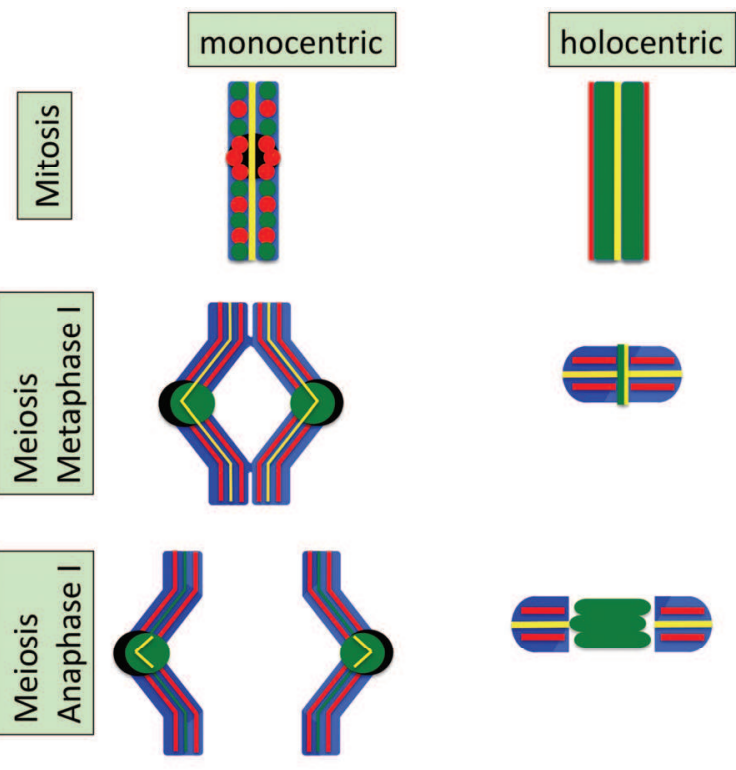

\begin{tabular}{|ll|}
\hline blue $=$ DNA & black $=$ centromere \\
green $=$ condensin I & red $=$ condensin II \\
yellow $=$ cohesin &
\end{tabular}

Fig. 4. The distribution of condensin I, condensin II, and cohesin on monocentric and holocentric chromosomes during mitosis and meiosis I.

In monocentric organisms, during prophase I of meiosis, cohesin molecules surrounding the site of crossover hold homologous chromosomes together until initiation of anaphase I. Homolog separation in meiosis I requires the resolution of sister chromatid cohesion distal to the chiasma. At the same time, sister chromatids must be held together until anaphase II by sister chromatid cohesion at the centromere. To achieve sequential loss of cohesin, in meiosis I arm cohesion is released, but centric cohesion is protected from degradation by Shugoshin/MEI-S322 (Kitajima et al., 2004; Resnick et al, 2006; Sakuno and Watanabe, 2009). This ensures that homologous chromosomes are no longer tethered, and can migrate to opposite poles, but sister chromatids travel together (Fig. 4) At anaphase II, centromeric cohesion is lost, and sister chromatids separate (Petronczki et al., 2003).

Holocentric organisms do not have a localized centromere, and phrases such as arm cohesion and centromeric cohesion do not apply. During C. elegans meiosis, the kinetochores form cuplike structures surrounding the entire volume of each homolog (Dumont et al., 2010) (See Fig. 2). Differentiation of domains within bivalents must therefore be coordinated in a different manner. Research in the last decade revealed that the formation of the crossover provides the primary clue for bivalent differentiation (Chan et al., 2004; Nabeshima et al., 2005). In C. elegans, each pair of homologs typically undergoes one crossover event in the terminal onethird of the chromosome. Toward the end of prophase I, bivalents are restructured into cross- 
shaped structures, in which the short arm is formed from the domain between the crossover and the closer chromosome end, and the long arm is formed from the domain between the crossover and the more distant chromosome end. Cohesin along the short arm is released in meiosis I to separate homologs, while cohesin along the long arm must be protected until anaphase II (reviewed in (Schvarzstein et al., 2010). Cohesion protection in C. elegans appears to be independent of Shugoshin, and instead is regulated by the HORMA domain containing protein HTP-1 and worm specific PP1 phosphatase interacting protein LAB-1 (de Carvalho et al., 2008; Martinez-Perez et al,. 2008). Note that while cohesion protection in meiosis I in monocentric organisms is performed at the centromere, in C. elegans cohesion is protected along the long arms of bivalents (Fig. 4).

As in monocentric organisms, distribution of chromosomal proteins differs between mitosis and meiosis in holocentric organisms as well. Our laboratory recently described the distinct distribution of condensin complexes in C. elegans, and compared their distributions in mitosis and meiosis (Collette et al., 2011). As in mouse oocytes, C. elegans condensin II occupies the core domain within each sister chromatid (Chan et al., 2004). Condensin I, on the other hand, is found at the midbivalent at the interface between homologs (Csankovszki et al., 2009; Collette et al., 2011) (Fig. 4).

\section{Holding sister together: Monopolin and Rec8}

Sisters are usually monooriented in meiosis I, even if homologs are not connected due to lack of chiasma formation. In most genetic backgrounds with defective crossover formation, homologs are not held together, but sister chromatids still segregate together toward the same pole (or are lost) during the first meiotic division. This finding implies that holding sisters together is not simply a consequence of keeping homologs apart. In budding yeast, a pair of sister kinetochores appears to be captured by a single microtubule (Winey et al., 2005). In higher eukaryotes, multiple microtubule attachments are made and both sister kinetochores are captured but they are connected to the same pole (Goldstein, 1981; Lee et al., 2000; Moore and Orr-Weaver, 1998; Parra et al., 2004.) Two possible mechanisms have been suggested to explain these findings: either sister kinetochores are fused into a single entity or one of the sisters is inactivated (Monje-Casas et al., 2007). Indeed, electron microscopy studies indicate that in meiosis I sister kinetochores are arranged side-by-side, as opposed to the back-to-back orientation seen in mitosis or meiosis II (Goldstein, 1981; Lee et al., 2000; Parra et al., 2004).

Studies of sister chromatid monoorientation in different model organisms have uncovered some interesting similarities and differences. Budding yeast makes use of a complex called monopolin to monoorient sisters. Monopolin is made up of Mam1, Csm1, Lrs4 and Hrr25 (Petronczki et al., 2003, 2006; Rabitsch et al., 2003; Toth et al., 2000). While in other model organisms the meiosis specific cohesin subunit Rec8 plays an important role in the process (see below), in budding yeast the function of Rec8 is not required for monoorientation (Toth et al., 2000, Yokobayashi et al., 2003), and monopolin appears to glue sisters together independent of cohesin function (Monje-Casas et al., 2007) On the other hand, condensin function is important for proper chromosome orientation, perhaps via correct localization of monopolin. In condensin depleted cells, monopolin association with kinetochores is reduced and a portion of sister kinetochores biorient (Brito et al., 2010). 
Monocentric organisms other than budding yeast do not use Monopolin for sister chromatid monoorienation, and instead make use of cohesin and other meiosis specific factors. Rec8 seems to play an especially important role. Rec8 deficiency in fission yeast and plants leads to biorientation of sister chromatids, resulting in equational rather than reductional division in meiosis I (Chelysheva et al., 2005; d'Erfurth et al., 2009; Watanabe and Nurse, 1999, Yu and Dawe, 2000). Replacement of Rec8 with its mitotic paralog also results in biorientation, indicating that $\operatorname{Rec} 8$ function is specifically required (Yokobayashi et al., 2003). While Rec8 is necessary for sister monoorientation, it is not sufficient (Watanabe and Nurse, 1999; Yokobayashi et al., 2003). In fission yeast at least one other meiosis specific factor, Moa1, assists Rec8 in the process (Yokobayashi and Watanabe, 2005). In fission yeast, Rec8 has a distinct localization pattern, and is also found at the central core region of the centromere, in addition to chromosome arms and pericentric regions (Yokobayashi et al., 2003). This is different from the localization pattern of mitotic cohesin, which is specifically reduced at the centromere core (see above). According to current models, cohesion at the core centromere induces sister kinetochore monorientation, and cohesion at pericentric regions (as in mitosis and meiosis II) allows kinetochores to move away from each other promoting biorientation (Sakuno et al., 2009; Sakuno and Watanabe, 2009).

Since holocentric organisms do not have a localized centromere, they must use a different method to hold sister chromatids together during meiosis I. Recent studies suggest that despite the difference in chromosomal organization, REC-8 plays an important role in sister monoorientation in C. elegans as well (Severson et al., 2009). In rec-8 mutant oocytes, homologs are not held together by a chiasma at metaphase due to an earlier defect in meiosis. However, rec- 8 mutant univalents (a pair of sister chromatids) become bioriented and separate from each other in meiosis I. Therefore $C$. elegans uses the same factor as monocentric organisms (REC-8), but this protein is functioning to promote sister monoorientation at different chromosomal domains: at the centromere in monocentric organisms and at the long arm of bivalents in C. elegans.

\section{Regulating kinetochore-microtubule attachments: Aurora B function promotes homolog biorientation}

As discussed above, in meiosis I homologous chromosomes become bioriented at the metaphase plate, as opposed to the biorientation of sister chromatids seen in mitosis and meiosis II. In principle, the problem to be solved is similar: tension must be established between two kinetochore entities that are connected. The difference is that this connection is centric cohesin in mitosis and chiasma between homologs during meiosis. During mitosis, Aurora B plays a crucial role in biorienting sister chromatids. During meiosis in fission yeast, budding yeast and mouse, Aurora B also localizes to the centromeric regions (Monje Casas et al., 2007; Parra et al., 2003, Petersen et al., 2001), therefore the protein is present at the right place and at the right time to regulate kinetochore-microtubule attachments in meiosis as well.

The first indication that Aurora B homologs are functioning in meiotic chromosome orientation came from studies in yeast. In budding yeast, Ipl1/Aurora B is needed for biorientation of homologs (Monje-Casas et al., 2007, Yu and Koshland, 2007). The results 
obtained in this system are consistent with the primary role of Aurora B to regulate turnover of kinetochore-microtubule attachments, similar to its role in mitosis. The fission yeast Aurora B homolog, Ark1, is also crucial for homolog biorientation during the first meiotic division (Hauf et al., 2007). Ark1 can promote biorientation of homologs, but it is also needed for biorientation of Rec8-deficient univalents (Hauf et al., 2007). In fact, Aurora B can promote biorientation even on kinetochores that are only loosely connected by a DNA thread (Dewar et al., 2004). These findings led to the suggestion that Aurora B can promote biorientation of any two kinetochores that are connected, further supporting the hypothesis that the molecular function of Aurora B is the same in mitosis and meiosis: correcting faulty kinetochore-microtubule attachments (Hauf et al., 2007).

Fission yeast Ark1 kinase appears to promote not only homolog biorientation, but also sister monoorientation. Ark1 can correct attachment of a unified pair of sister chromatids to both poles (merotelic attachment). While corrections of these merotelic attachments ultimately promote sister monoorientation, this mechanism is fundamentally different from the mechanism used by Rec 8 and Moa1 to alter kinetochore geometry discussed above (Hauf et al., 2007).

On the holocentric chromosomes of C. elegans, Aurora B/AIR-2 is also located in an ideal position for regulating chromosome orientation, although this role has not been formally demonstrated yet. When bivalents orient at the metaphase plate, their long arms are parallel to spindle microtubules, and the midbivalent domains line up at the metaphase plate. Surprisingly, microtubule density on the poleward end of bivalents is low. Instead chromosomes are ensheathed by lateral microtubule bundles (Wignall and Willeneuve, 2009) (See Fig. 4). This ensheathment by lateral microtubules naturally promotes the orientation of homologs away from each other (Schwarzstein et al., 2010). Importantly, Aurora B/AIR-2 is found at the midbivalent domain, at the homolog interface (Kaitna et al., 2002; Rogers et al., 2002). Aurora B is perfectly situated here to promote homolog biorientation by performing its usual function: destabilizing incorrectly attached microtubules. Ensuring that microtubules do not attach at the zone of high Aurora B activity, coupled with ensheathment of bivalents by lateral microtubules provides an attractive model for biorientation of holocentric homologs.

\section{Regulating chromosome orientation: a role for condensin?}

Condensin I during mouse (Lee et al., 2011) and C. elegans meiosis (Collette et al., 2011) colocalizes with Aurora B. In the mouse, both condensin I and Aurora B are enriched at the centromere during metaphase $\mathrm{I}-$ at the domain important for regulating chromosome orientation. We recently demonstrated that in C. elegans AIR-2/Aurora B and condensin I colocalize at metaphase and anaphase of both meiotic divisions. Interestingly, condensin I occupies the same domain as AIR-2 not just on chromosomes at metaphase I and II, but also on the spindle between separating chromosomes at anaphase (Fig. 5). In addition, we also showed that correct targeting of condensin I to the midbivalent in meiosis I is dependent on AIR-2 (Collette et al., 2011). These results raise the interesting possibility that condensin I may aid Aurora B in performing its functions in regulating chromosome orientation in meiosis. 


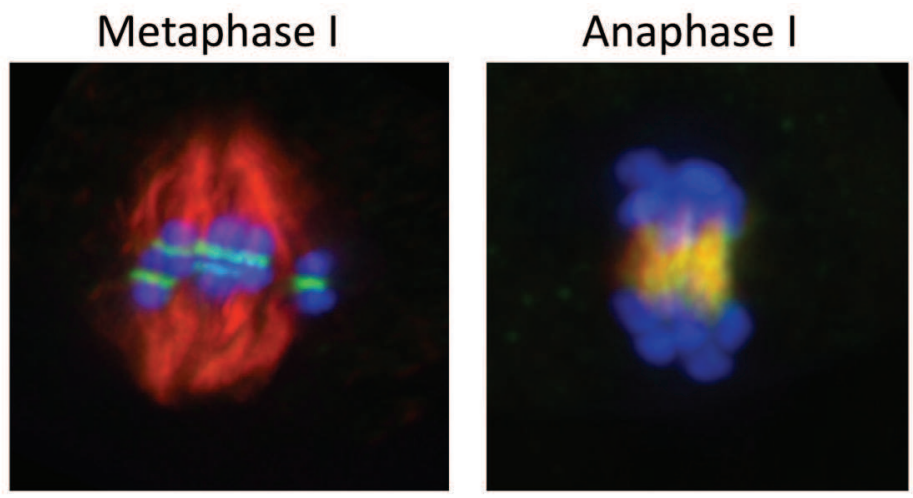

Fig. 5. Fluorescence microscopy images of condensin I distribution during C. elegans meiosis. During metaphase I, condensin I associates with the midbivalent domain, and during anaphase it is found on spindle microtubules between separating chromosomes. Microtubules are shown in red, condensin I in green, and chromosomes in blue.

Condensin depletion experiments support a role for condensin in regulating chromosome orientation. In budding yeast, condensin depletion leads to reduced localization of Monopolin subunit Mam1 to kinetochores and increased incidence of sister chromatid biorientation (Brito et al., 2010). In mouse oocytes, injections of SMC2 antibodies (which interfere with the function of both condensin complexes) led to a portion of sister chromatids assuming back-to-back, rather than side-by-side orientation. Anti-CAP-H injection (which interferes with condensin I only) caused disorganized centromeres and chromosome alignment problems (Lee et al., 2011). Condensin function may influence kinetochore structure, or may affect the underlying structure of centromeric chromatin (Bernad et al., 2011; Lee et al., 2011; Ono et al., 2004). Whether similar defects will be seen upon condensin I depletion in holocentric organisms as well remains to be demonstrated. However, given the colocalization of Aurora B and condensin I in mouse and C. elegans meiosis, the role for Aurora B in chromosome orientation in yeast and mouse meiosis, and the apparently conserved function of Aurora B in different organisms, we would like to propose that condensin I may aid chromosome orientation in C. elegans meiosis as well.

\section{Conclusion}

During meiosis in monocentric organisms, the centromere is used as a site where kinetochore-microtubule attachments, and therefore chromosome orientation, are regulated. In organisms other than budding yeast, enrichment of meiotic Rec8-containing cohesin at core centromeric regions promote monoorientation of sister chromatids. Aurora B at centromeric region appears to function to correct aberrant kinetochore-microtubule attachments to promote biorientation of homologs and prevent merotelic attachment of unified sister kinetochores. During meiosis in C. elegans, the site of the crossover, and not a localized centromere, ultimately determines the plane of chromosome orientation. During metaphase, the short arms of bivalents are lined up along the metaphase plate and the long arms point toward opposite poles. The kinetochore "cups" the entire volume of sister chromatids along the long arms. The activities that monoorient sisters and biorient 
homologs are concentrated at opposite domains of the bivalents. REC-8 promotes sister chromatid monoorientation along the long arms. By contrast, Aurora B is restricted to the short arm, where by preventing microtubules from "crossing the plane" of the bivalent short arm, it can ensure that sisters stay together, and homologs stay apart.

One way to conceptualize the differences between holocentric and monocentric chromosome orientation in meiosis is to think of holocentric bivalents as one large centromere without extended chromosome arms. The entire holocentric chromosome is cupped by the kinetochore. One domain within the kinetochore-bound domain (the long arm) contains REC-8, and according to this analogy, is comparable to core centromeric regions of monocentric chromosomes. Another domain (the short arm) is comparable to regions of Aurora B enrichment at centromeres. The organization of holocentric chromosomes magnifies the distinction between the various specialized chromosomal domains and makes it an ideal setting in which to examine these differences.

\section{References}

Andrews, P. D., Y. Ovechkina, et al. (2004). Aurora B regulates MCAK at the mitotic centromere. Dev Cell 6(2): 253-268.

Bernad, R., P. Sanchez, et al. (2011). Xenopus HJURP and condensin II are required for CENP-A assembly. J Cell Biol 192(4): 569-582.

Bernard, P., J. F. Maure, et al. (2001). Requirement of heterochromatin for cohesion at centromeres. Science 294(5551): 2539-2542.

Bhat, M. A., A. V. Philp, et al. (1996). Chromatid segregation at anaphase requires the barren product, a novel chromosome-associated protein that interacts with Topoisomerase II. Cell 87(6): 1103-1114.

Biggins, S. and A. W. Murray (2001). The budding yeast protein kinase Ipl1/Aurora allows the absence of tension to activate the spindle checkpoint. Genes Dev 15(23): 31183129 .

Biggins, S., F. F. Severin, et al. (1999). The conserved protein kinase Ipl1 regulates microtubule binding to kinetochores in budding yeast. Genes Dev 13(5): 532-544.

Brito, I. L., H. G. Yu, et al. (2010). Condensins promote coorientation of sister chromatids during meiosis I in budding yeast. Genetics 185(1): 55-64.

Carvalho, A., M. Carmena, et al. (2003). Survivin is required for stable checkpoint activation in taxol-treated HeLa cells. J Cell Sci 116(Pt 14): 2987-2998.

Chan, R. C., A. F. Severson, et al. (2004). Condensin restructures chromosomes in preparation for meiotic divisions. J Cell Biol 167(4): 613-625.

Cheeseman, I. M., S. Anderson, et al. (2002). Phospho-regulation of kinetochore-microtubule attachments by the Aurora kinase Ipl1p. Cell 111(2): 163-172.

Cheeseman, I. M., J. S. Chappie, et al. (2006). The conserved KMN network constitutes the core microtubule-binding site of the kinetochore. Cell 127(5): 983-997.

Chelysheva, L., S. Diallo, et al. (2005). AtREC8 and AtSCC3 are essential to the monopolar orientation of the kinetochores during meiosis. J Cell Sci 118(Pt 20): 4621-4632.

Ciferri, C., S. Pasqualato, et al. (2008). Implications for kinetochore-microtubule attachment from the structure of an engineered Ndc80 complex. Cell 133(3): 427-439.

Cimini, D. (2007). Detection and correction of merotelic kinetochore orientation by Aurora B and its partners. Cell Cycle 6(13): 1558-1564. 
Cimini, D., X. Wan, et al. (2006). Aurora kinase promotes turnover of kinetochore microtubules to reduce chromosome segregation errors. Curr Biol 16(17): 1711-1718.

Cobbe, N., E. Savvidou, et al. (2006). Diverse mitotic and interphase functions of condensins in Drosophila. Genetics 172(2): 991-1008.

Coelho, P. A., J. Queiroz-Machado, et al. (2003). Condensin-dependent localisation of topoisomerase II to an axial chromosomal structure is required for sister chromatid resolution during mitosis. J Cell Sci 116(Pt 23): 4763-4776.

Collette, K. S., Petty, E. L., et al., Different roles for Aurora B in condensin targeting during mitosis and meiosis. (2011). J Cell Sci: in press

Csankovszki, G., K. Collette, et al. (2009). Three distinct condensin complexes control C. elegans chromosome dynamics. Curr Biol 19(1): 9-19.

d'Erfurth, I., S. Jolivet, et al. (2009). Turning meiosis into mitosis. PLoS Biol 7(6): e1000124.

de Carvalho, C. E., S. Zaaijer, et al. (2008). LAB-1 antagonizes the Aurora B kinase in C. elegans. Genes Dev 22(20): 2869-2885.

Dej, K. J., C. Ahn, et al. (2004). Mutations in the Drosophila condensin subunit dCAP-G: defining the role of condensin for chromosome condensation in mitosis and gene expression in interphase. Genetics 168(2): 895-906.

DeLuca, J. G., W. E. Gall, et al. (2006). Kinetochore microtubule dynamics and attachment stability are regulated by Hec1. Cell 127(5): 969-982.

Dewar, H., K. Tanaka, et al. (2004). Tension between two kinetochores suffices for their biorientation on the mitotic spindle. Nature 428(6978): 93-97.

Ditchfield, C., V. L. Johnson, et al. (2003). Aurora B couples chromosome alignment with anaphase by targeting BubR1, Mad2, and Cenp-E to kinetochores. LCell Biol 161(2): 267-280.

Dumont, J., K. Oegema, et al. (2010). A kinetochore-independent mechanism drives anaphase chromosome separation during acentrosomal meiosis. Nat Cell Biol 12(9): 894-901.

Gerlich, D., T. Hirota, et al. (2006). Condensin I stabilizes chromosomes mechanically through a dynamic interaction in live cells. Curr Biol 16(4): 333-344.

Goldstein, L. S. (1981). Kinetochore structure and its role in chromosome orientation during the first meiotic division in male D. melanogaster. Cell 25(3): 591-602.

Hagstrom, K. A., V. F. Holmes, et al. (2002). C. elegans condensin promotes mitotic chromosome architecture, centromere organization, and sister chromatid segregation during mitosis and meiosis. Genes Dev 16(6): 729-742.

Hartl, T. A., S. J. Sweeney, et al. (2008). Condensin II resolves chromosomal associations to enable anaphase I segregation in Drosophila male meiosis. PLoS Genet 4(10): e1000228.

Hauf, S., A. Biswas, et al. (2007). Aurora controls sister kinetochore mono-orientation and homolog bi-orientation in meiosis-I. EMBO J 26(21): 4475-4486.

Hauf, S., R. W. Cole, et al. (2003). The small molecule Hesperadin reveals a role for Aurora B in correcting kinetochore-microtubule attachment and in maintaining the spindle assembly checkpoint. J Cell Biol 161(2): 281-294.

Hauf, S. \& Watanabe, Y. (2004). Kinetochore Orientation in Mitosis and Meiosis. Cell 119:317-327.

Hirano, T. (2000). Chromosome cohesion, condensation, and separation. Annu Rev Biochem 69: 115-144. 
Hirano, T. (2002). The ABCs of SMC proteins: two-armed ATPases for chromosome condensation, cohesion, and repair. Genes Dev 16(4): 399-414.

Hirano, T. (2004). Chromosome shaping by two condensins. Cell Cycle 3(1): 26-28.

Hirano, T. and T. J. Mitchison (1994). A heterodimeric coiled-coil protein required for mitotic chromosome condensation in vitro. Cell 79(3): 449-458.

Hirota, T., D. Gerlich, et al. (2004). Distinct functions of condensin I and II in mitotic chromosome assembly. J Cell Sci 117(26): 6435-6445.

Honda, R., R. Korner, et al. (2003). Exploring the functional interactions between Aurora B, INCENP, and survivin in mitosis. Mol Biol Cell 14(8): 3325-3341.

Hudson, D. F., K. M. Marshall, et al. (2009). Condensin: Architect of mitotic chromosomes. Chromosome Res 17(2): 131-144.

Hudson, D. F., P. Vagnarelli, et al. (2003). Condensin is required for nonhistone protein assembly and structural integrity of vertebrate mitotic chromosomes. Dev Cell 5(2): 323-336.

Jessberger, R. (2002). The many functions of SMC proteins in chromosome dynamics. Nat Rev Mol Cell Biol 3(10): 767-778.

Kaitna, S., P. Pasierbek, et al. (2002). The aurora B kinase AIR-2 regulates kinetochores during mitosis and is required for separation of homologous Chromosomes during meiosis. Curr Biol 12(10): 798-812.

Kallio, M. J., M. L. McCleland, et al. (2002). Inhibition of aurora B kinase blocks chromosome segregation, overrides the spindle checkpoint, and perturbs microtubule dynamics in mitosis. Curr Biol 12(11): 900-905.

Kitajima, T. S., Kawashima, S. A. et al. (2004). The conserved kinetochore protein shugoshin protects centromeric cohesion during meiosis. Nature 427: 510-517.

Knowlton, A. L., W. Lan, et al. (2006). Aurora B is enriched at merotelic attachment sites, where it regulates MCAK. Curr Biol 16(17): 1705-1710.

Lampson, M. A., K. Renduchitala, et al. (2004). Correcting improper chromosome-spindle attachments during cell division. Nat Cell Biol 6(3): 232-237.

Lan, W., X. Zhang, et al. (2004). Aurora B phosphorylates centromeric MCAK and regulates its localization and microtubule depolymerization activity. Curr Biol 14(4): 273-286.

Lee, J., T. Miyano, et al. (2000). Specific regulation of CENP-E and kinetochores during meiosis I/meiosis II transition in pig oocytes. Mol Reprod Dev 56(1): 51-62.

Lee, J., S. Ogushi, et al. (2011). Condensin I and II are essential for construction of bivalent chromosomes in mouse oocytes. Mol Biol Cell.

Lens, S. M., R. M. Wolthuis, et al. (2003). Survivin is required for a sustained spindle checkpoint arrest in response to lack of tension. EMBO J 22(12): 2934-2947.

Lieb, J. D., M. R. Albrecht, et al. (1998). MIX-1: an essential component of the C. elegans mitotic machinery executes X chromosome dosage compensation. Cell 92(2): 265277.

Liu, D., G. Vader, et al. (2009). Sensing chromosome bi-orientation by spatial separation of aurora B kinase from kinetochore substrates. Science 323(5919): 1350-1353.

Losada, A. and T. Hirano (2005). Dynamic molecular linkers of the genome: the first decade of SMC proteins. Genes Dev 19(11): 1269-1287.

Marston, A. L. and A. Amon (2004). Meiosis: cell-cycle controls shuffle and deal. Nat Rev Mol Cell Biol 5(12): 983-997. 
Martinez-Perez, E., M. Schvarzstein, et al. (2008). Crossovers trigger a remodeling of meiotic chromosome axis composition that is linked to two-step loss of sister chromatid cohesion. Genes Dev 22(20): 2886-2901.

Monje-Casas, F., V. R. Prabhu, et al. (2007). Kinetochore orientation during meiosis is controlled by Aurora B and the monopolin complex. Cell 128(3): 477-490.

Moore, D. P. and T. L. Orr-Weaver (1998). Chromosome segregation during meiosis: building an unambivalent bivalent. Curr Top Dev Biol 37: 263-299.

Nabeshima, K., A. M. Villeneuve, et al. (2005). Crossing over is coupled to late meiotic prophase bivalent differentiation through asymmetric disassembly of the SC. J Cell Biol 168(5): 683-689.

Nasmyth, K. (2002). Segregating sister genomes: the molecular biology of chromosome separation. Science 297(5581): 559-565.

Nasmyth, K., J. M. Peters, et al. (2001). Splitting the chromosome: cutting the ties that bind sister chromatids. Novartis Found Symp 237: 113-133; discussion 133-118, 158-163.

Nonaka, N., T. Kitajima, et al. (2002). Recruitment of cohesin to heterochromatic regions by Swi6/HP1 in fission yeast. Nat Cell Biol 4(1): 89-93.

Oliveira, R. A., P. A. Coelho, et al. (2005). The condensin I subunit Barren/CAP-H is essential for the structural integrity of centromeric heterochromatin during mitosis. Mol Cell Biol 25(20): 8971-8984.

Ono, T., Y. Fang, et al. (2004). Spatial and temporal regulation of Condensins I and II in mitotic chromosome assembly in human cells. Mol Biol Cell 15(7): 3296-3308.

Ono, T., A. Losada, et al. (2003). Differential contributions of condensin I and condensin II to mitotic chromosome architecture in vertebrate cells. Cell 115(1): 109-121.

Page, S. L. \& Hawley, R. S. (2003). Chromosome choreography: The meiotic ballet. Science 310: 785-789.

Paliulis, L. V. and R. B. Nicklas (2000). The reduction of chromosome number in meiosis is determined by properties built into the chromosomes. J Cell Biol 150(6): 1223-1232.

Parra, M. T., A. Viera, et al. (2004). Involvement of the cohesin Rad21 and SCP3 in monopolar attachment of sister kinetochores during mouse meiosis I. J Cell Sci 117(Pt 7): 1221-1234.

Parra, M. T., A. Viera, et al. (2003). Dynamic relocalization of the chromosomal passenger complex proteins inner centromere protein (INCENP) and aurora-B kinase during male mouse meiosis. J Cell Sci 116(Pt 6): 961-974.

Petersen, J., J. Paris, et al. (2001). The S. pombe aurora-related kinase Ark1 associates with mitotic structures in a stage dependent manner and is required for chromosome segregation. J Cell Sci 114(Pt 24): 4371-4384.

Petronczki, M., J. Matos, et al. (2006). Monopolar attachment of sister kinetochores at meiosis I requires casein kinase 1. Cell 126(6): 1049-1064.

Petronczki, M., M. F. Siomos, et al. (2003). Un menage a quatre: the molecular biology of chromosome segregation in meiosis. Cell 112(4): 423-440.

Rabitsch, K. P., M. Petronczki, et al. (2003). Kinetochore recruitment of two nucleolar proteins is required for homolog segregation in meiosis I. Dev Cell 4(4): 535-548.

Resnick, T. D., Satinover, D. L. et al., (2006) INCENP and Aurora B promote meiotic sister chromatid cohesion through localization of the Shugoshin MEI-S322 in Drosophila. Dev Cell 11: 57-68. 
Ribeiro, S. A., J. C. Gatlin, et al. (2009). Condensin regulates the stiffness of vertebrate centromeres. Mol Biol Cell 20(9): 2371-2380.

Rogers, E., J. D. Bishop, et al. (2002). The aurora kinase AIR-2 functions in the release of chromosome cohesion in Caenorhabditis elegans meiosis. J Cell Biol 157(2): 219-229.

Ruchaud, S., M. Carmena, et al. (2007). Chromosomal passengers: conducting cell division. Nat Rev Mol Cell Biol 8(10): 798-812.

Saitoh, N., I. G. Goldberg, et al. (1994). ScII: an abundant chromosome scaffold protein is a member of a family of putative ATPases with an unusual predicted tertiary structure. J Cell Biol 127(2): 303-318.

Saka, Y., T. Sutani, et al. (1994). Fission yeast cut3 and cut14, members of a ubiquitous protein family, are required for chromosome condensation and segregation in mitosis. EMBO J 13(20): 4938-4952.

Sakuno, T., K. Tada, et al. (2009). Kinetochore geometry defined by cohesion within the centromere. Nature 458(7240): 852-858.

Sakuno, T. K. \& Watanabe Y. (2009) Studies of meiosis disclose distinct roles of cohesion in the core centromere and pericentric regions. Chromosome Res 17:239-249.

Samoshkin, A., A. Arnaoutov, et al. (2009). Human condensin function is essential for centromeric chromatin assembly and proper sister kinetochore orientation. PLoS ONE 4(8): e6831.

Sandall, S., F. Severin, et al. (2006). A Bir1-Sli15 complex connects centromeres to microtubules and is required to sense kinetochore tension. Cell 127(6): 1179-1191.

Savvidou, E., N. Cobbe, et al. (2005). Drosophila CAP-D2 is required for condensin complex stability and resolution of sister chromatids. J Cell Sci 118(Pt 11): 2529-2543.

Schumacher, J. M., A. Golden, et al. (1998). AIR-2: An Aurora/Ipl1-related protein kinase associated with chromosomes and midbody microtubules is required for polar body extrusion and cytokinesis in Caenorhabditis elegans embryos. J Cell Biol 143(6): 1635-1646.

Schvarzstein, M., S. M. Wignall, et al. (2010). Coordinating cohesion, co-orientation, and congression during meiosis: lessons from holocentric chromosomes. Genes Dev 24(3): 219-228.

Severson, A. F., L. Ling, et al. (2009). The axial element protein HTP-3 promotes cohesin loading and meiotic axis assembly in C. elegans to implement the meiotic program of chromosome segregation. Genes Dev 23(15): 1763-1778.

Shang, C., T. R. Hazbun, et al. (2003). Kinetochore protein interactions and their regulation by the Aurora kinase Ipl1p. Mol Biol Cell 14(8): 3342-3355.

Siddiqui, N. U., P. E. Stronghill, et al. (2003). Mutations in Arabidopsis condensin genes disrupt embryogenesis, meristem organization and segregation of homologous chromosomes during meiosis. Development 130(14): 3283-3295.

Sonoda, E., T. Matsusaka, et al. (2001). Scc1/Rad21/Mcd1 is required for sister chromatid cohesion and kinetochore function in vertebrate cells. Dev Cell 1(6): 759-770.

Stear, J. H. and M. B. Roth (2002). Characterization of HCP-6, a C. elegans protein required to prevent chromosome twisting and merotelic attachment. Genes Dev 16(12): 14981508.

Steffensen, S., P. A. Coelho, et al. (2001). A role for Drosophila SMC4 in the resolution of sister chromatids in mitosis. Curr Biol 11(5): 295-307. 
Strunnikov, A. V., E. Hogan, et al. (1995). SMC2, a Saccharomyces cerevisiae gene essential for chromosome segregation and condensation, defines a subgroup within the SMC family. Genes Dev 9(5): 587-599.

Tanaka, T., J. Fuchs, et al. (2000). Cohesin ensures bipolar attachment of microtubules to sister centromeres and resists their precocious separation. Nat Cell Biol 2(8): 492-499.

Tanaka, T. U. (2002). Bi-orienting chromosomes on the mitotic spindle. Curr Opin Cell Biol 14(3): 365-371.

Tanaka, T. U., N. Rachidi, et al. (2002). Evidence that the Ipl1-Sli15 (Aurora kinase-INCENP) complex promotes chromosome bi-orientation by altering kinetochore-spindle pole connections. Cell 108(3): 317-329.

Tomonaga, T., K. Nagao, et al. (2000). Characterization of fission yeast cohesin: essential anaphase proteolysis of Rad21 phosphorylated in the S phase. Genes Dev 14(21): 2757-2770.

Toth, A., K. P. Rabitsch, et al. (2000). Functional genomics identifies monopolin: a kinetochore protein required for segregation of homologs during meiosis i. Cell 103(7): 1155-1168.

Vagnarelli, P. and W. C. Earnshaw (2004). Chromosomal passengers: the four-dimensional regulation of mitotic events. Chromosoma 113(5): 211-222.

Vagnarelli, P., D. F. Hudson, et al. (2006). Condensin and Repo-Man-PP1 co-operate in the regulation of chromosome architecture during mitosis. Nat Cell Biol 8(10): 11331142.

Watanabe, Y. (2010). Temporal and spatial regulation of targeting aurora B to the inner centromere. Cold Spring Harb Symp Quant Biol 75: 419-423.

Watanabe, Y. and P. Nurse (1999). Cohesin Rec8 is required for reductional chromosome segregation at meiosis. Nature 400(6743): 461-464.

Watrin, E. and V. Legagneux (2005). Contribution of hCAP-D2, a non-SMC subunit of condensin I, to chromosome and chromosomal protein dynamics during mitosis. Mol Cell Biol 25(2): 740-750.

Wignall, S. M., R. Deehan, et al. (2003). The condensin complex is required for proper spindle assembly and chromosome segregation in Xenopus egg extracts. J Cell Biol 161(6): 1041-1051.

Wignall, S. M. and A. M. Villeneuve (2009). Lateral microtubule bundles promote chromosome alignment during acentrosomal oocyte meiosis. Nat Cell Biol 11(7): 839-844.

Winey, M., G. P. Morgan, et al. (2005). Three-dimensional ultrastructure of Saccharomyces cerevisiae meiotic spindles. Mol Biol Cell 16(3): 1178-1188.

Yokobayashi, S. and Y. Watanabe (2005). The kinetochore protein Moa1 enables cohesionmediated monopolar attachment at meiosis I. Cell 123(5): 803-817.

Yokobayashi, S., M. Yamamoto, et al. (2003). Cohesins determine the attachment manner of kinetochores to spindle microtubules at meiosis I in fission yeast. Mol Cell Biol 23(11): 3965-3973.

$\mathrm{Yu}, \mathrm{H}$. G. and R. K. Dawe (2000). Functional redundancy in the maize meiotic kinetochore. J Cell Biol 151(1): 131-142.

$\mathrm{Yu}$, H. G. and D. Koshland (2005). Chromosome morphogenesis: condensin-dependent cohesin removal during meiosis. Cell 123(3): 397-407. 
Yu, H. G. and D. Koshland (2007). The Aurora kinase Ipl1 maintains the centromeric localization of PP2A to protect cohesin during meiosis. J Cell Biol 176(7): 911-918.

$\mathrm{Yu}, \mathrm{H}$. G. and D. E. Koshland (2003). Meiotic condensin is required for proper chromosome compaction, SC assembly, and resolution of recombination-dependent chromosome linkages. J Cell Biol 163(5): 937-947.

Zhu, C., E. Bossy-Wetzel, et al. (2005). Recruitment of MKLP1 to the spindle midzone/midbody by INCENP is essential for midbody formation and completion of cytokinesis in human cells. Biochem J 389(Pt 2): 373-381. 


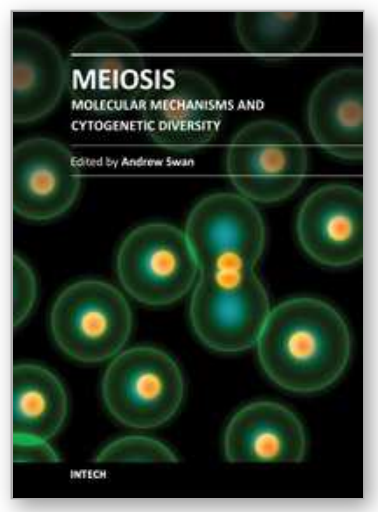

\author{
Meiosis - Molecular Mechanisms and Cytogenetic Diversity \\ Edited by Dr. Andrew Swan
}

ISBN 978-953-51-0118-5

Hard cover, 472 pages

Publisher InTech

Published online 29, February, 2012

Published in print edition February, 2012

Meiosis, the process of forming gametes in preparation for sexual reproduction, has long been a focus of intense study. Meiosis has been studied at the cytological, genetic, molecular and cellular levels. Studies in model systems have revealed common underlying mechanisms while in parallel, studies in diverse organisms have revealed the incredible variation in meiotic mechanisms. This book brings together many of the diverse strands of investigation into this fascinating and challenging field of biology.

\title{
How to reference
}

In order to correctly reference this scholarly work, feel free to copy and paste the following:

Karishma Collette and Györgyi Csankovszki (2012). Facing the Correct Pole: The Challenge of Orienting Chromosomes for Meiotic Divisions, Meiosis - Molecular Mechanisms and Cytogenetic Diversity, Dr. Andrew Swan (Ed.), ISBN: 978-953-51-0118-5, InTech, Available from: http://www.intechopen.com/books/meiosismolecular-mechanisms-and-cytogenetic-diversity/facing-the-correct-pole-the-challenge-of-orientingchromosomes-for-meiotic-divisions

\section{INTECH}

open science | open minds

\section{InTech Europe}

University Campus STeP Ri

Slavka Krautzeka 83/A

51000 Rijeka, Croatia

Phone: +385 (51) 770447

Fax: +385 (51) 686166

www.intechopen.com

\section{InTech China}

Unit 405, Office Block, Hotel Equatorial Shanghai

No.65, Yan An Road (West), Shanghai, 200040, China

中国上海市延安西路65号上海国际贵都大饭店办公楼 405 单元

Phone: +86-21-62489820

Fax: +86-21-62489821 
(C) 2012 The Author(s). Licensee IntechOpen. This is an open access article distributed under the terms of the Creative Commons Attribution 3.0 License, which permits unrestricted use, distribution, and reproduction in any medium, provided the original work is properly cited. 